\title{
Variability in Saturn's bow shock and magnetopause from Pioneer and Voyager: Probabilistic predictions and initial observations by Cassini
}

\author{
S. Hendricks ${ }^{1}$ and F. M. Neubauer \\ Institute for Geophysics and Meteorology, University of Cologne, Cologne, Germany \\ M. K. Dougherty and N. Achilleos \\ Space and Atmospheric Physics, Blackett Laboratory, Imperial College London, London, UK \\ C. T. Russell \\ Institute of Geophysics and Planetary Physics, University of California, Los Angeles, California, USA \\ Received 27 January 2005; revised 9 June 2005; accepted 23 June 2005; published 4 August 2005.
}

[1] Probability distributions for the location of the Saturnian bow shock and magnetopause have been derived by extrapolating observations of dynamic solar wind pressures to the position of Saturn's orbit. These observations are those made by the Pioneer 11, Voyager 1 and 2 spacecraft near Saturn's orbit and by the Ulysses spacecraft near its aphelion. The magnetopause subsolar distance (measured from Saturn's center) is obtained using pressure equilibrium. The bow shock standoff distance is determined using empirical relations between bow shock size and solar wind dynamic pressure. Simple 2-D geometric models of the magnetopause and bow shock surfaces have been used to determine their morphologies over a large range in local time. Three cases have been studied: (1) An Earth-type magnetosphere with low internal plasma pressure; (2) An intermediate case calibrated with Voyager 1 observations; and (3) A Jupiter-like inflated magnetosphere. The comparison of these models with initial observations from the initial sunward orbits of the Cassini spacecraft indicates a more inflated magnetosphere than postulated by the previous modelling of the PioneerVoyager encounters. Citation: Hendricks, S., F. M. Neubauer, M. K. Dougherty, N. Achilleos, and C. T. Russell (2005), Variability in Saturn's bow shock and magnetopause from Pioneer and Voyager: Probabilistic predictions and initial observations by Cassini, Geophys. Res. Lett., 32, L20S08, doi:10.1029/ 2005 GL022569.

\section{Introduction}

[2] Considerable temporal variability in the size of the Saturnian magnetopause and bow shock has been observed during the encounters with Saturn of the Pioneer 11 (P11) and both Voyager spacecraft (V1 and V2). This variability contributes to the wide range in plasma conditions at the locations of Saturn's outer satellites. In particular the plasma environment of Titan is highly sensitive to the size of the bow shock and magnetopause boundaries. This is because the arc of Titan's orbit near Saturn local noon is situated very close (within a few Saturn radii) to the mean position

\footnotetext{
${ }^{1}$ Now at Alfred-Wegener Institute for Polar and Marine Sciences, Bremerhaven, Germany.
}

Copyright 2005 by the American Geophysical Union. 0094-8276/05/2005GL022569 of Saturn's magnetopause. A study of the interaction between Titan and the three distinct types of plasma in which it may be immersed - magnetosphere, magnetosheath, and solar wind - has been described by Wolf and Neubauer [1982]. An earlier analysis of the magnetopause and bow shock observations from the Voyager and Pioneer encounters has been carried out by Slavin et al. [1985]. The results show that for mean conditions of the solar wind Titan is outside the magnetosphere when it is located at local noon with respect to Saturn. In addition, the statistical probability of Titan being situated upstream of Saturn's bow shock is very low.

[3] The purpose of this work is to estimate the bow shock and magnetopause locations based on a probabilistic model, with application to the Cassini Titan flybys. We use a simple parametric approach for the boundary distances to avoid 3-D magnetohydrodynamic (MHD) calculations. Since the end of this study all flybys of Titan near Saturn local noon (V1, Cassini TA [Backes et al., 2005], TB, TC, T3) took place in the magnetosphere. Recently developed models of the magnetosphere shape are used to make an independent validation of the Cassini observations.

\section{Method}

[4] A detailed review of the Pioneer-Voyager observations has been given by Behannon et al. [1983]. Because all spacecraft entered the Saturnian system in the noon sector, each of these three flybys represents a possibility for testing the pressure balance conditions at the subsolar point. For proper calibration of the resulting model the solar wind conditions during the encounter must be taken into account. During the P11 and V2 flybys, dramatic changes in solar wind conditions were observed. To explain the unusually expanded magnetosphere observed by V2, Saturn's interaction with the Jovian magnetotail has been considered [Behannon et al., 1983; Scarf et al., 1981]. During the P11 encounter, changes of the solar wind parameters have also been reported [Wolfe et al., 1980]. A feature of the near-local-noon observations of both V2 and P11 is a lack of multiple crossings of the magnetopause in a short period of time. These observations indicate a fast moving boundary, since multiple detections are expected for small oscillations about a state of equilibrium, or effects of surface waves. These multiple observations had been detected by 
Table 1. Calibration Model Overview ${ }^{\mathrm{a}}$

\begin{tabular}{lccc}
\hline \multicolumn{1}{c}{$i$} & Model 1 & Model 2 & Model 3 \\
\hline$p_{\text {dyn }}, \mathrm{Pa}$ & $7.84 \cdot 10^{-12}$ & $3.52 \cdot 10^{-11}$ & $6.27 \cdot 10^{-11}$ \\
$f$ & 2.32 & 2.32 & - \\
$f_{p}$ & 1 & 2 & - \\
$f_{r}$ & 1 & 1.5 & - \\
$C_{i}$ & 6.11 & 27.49 & 48.88 \\
\hline
\end{tabular}

${ }^{\mathrm{a}}$ Last measured solar wind dynamic pressure: $3.1 \cdot 10^{-11} \mathrm{~Pa}$. Subsolar magnetopause distance: $r_{m p, 0}=22.7 R_{S}(\mathrm{~V} 1)$

V1 in a short interval [Ness et al., 1981]; hence a stable location of the magnetopause can be assumed for this encounter. Furthermore it was noted that the solar wind was quite stable during the V1 encounter [Ness et al., 1981]. Based upon these interpretations, the V1 magnetopause observation seems to be most appropriate for linking a magnetopause observation to solar wind conditions.

[5] Because of the insufficient knowledge of the external conditions during the V1 encounter three cases have been employed in this study. Comparable models for both magnetopause and bow shock [Slavin et al., 1983, 1985] exist. For each of the calibration models the subsolar standoff distance of the magnetopause and bow shock have been determined. It is assumed that both magnetopause and bow shock have rotational symmetry around the Saturn-Sun direction and that their shapes are mainly controlled by the subsolar standoff distance.

\subsection{Magnetopause Models}

[6] The magnetopause is treated as an ideal tangential discontinuity, with a balance between total (magnetic plus plasma) pressure on either side. Since there is no analytical method to describe the total external pressure at the stagnation point for arbitrary solar wind plasma and magnetic parameters [Petrinec and Russell, 1997], a gasdynamic approach is used. As a widespread method, the external pressure on the stagnation point can be written as a function of the solar wind dynamic pressure $P_{e}=k p_{d y n}$ [Spreiter et al., 1966]. The value for $k$ reaches 0.881 for high Mach numbers, which are appropriate at Saturn's heliographic distance.

[7] For the calculation of the internal pressure four components are taken into account: 1) Saturn's internal magnetic field, 2) the Chapman-Ferraro (CF) currents in the magnetopause, 3 ) the magnetospheric ring current, and 4) the plasma pressure in the outer magnetosphere.

[8] Saturn's internal field is assumed to be that of a dipole whose axis of symmetry is aligned with the planet's axis of rotation. The stagnation point of the magnetosheath flow deviates from the equatorial plane in a range from $\psi=$ $-26.73^{\circ}$ to $26.73^{\circ}$ for a complete orbit of Saturn. The dipole field strength should vary with $\cos \psi$ at the subsolar location [Voigt, 1995]. For the primary Cassini mission an average flow angle of $\psi=-15.6^{\circ}$ is chosen, which represents the median of the interval from 2004 to 2008. The CF currents amplify the strength of the dipole field inside the magnetopause by more than a factor of two $(f=$ 2.32 [Voigt, 1995]).

[9] The influence of the ring current is described by a constant ratio between the dipole field $B_{d p}$ and the ring current field $B_{r c}$ at the magnetopause $\left(f_{r}=1+B_{r c} / B_{d p}\right)$ neglecting dynamical effects. The ratio is determined from the Connerney et al. [1983] model by a Biot-Savart inte- gration of the current distribution. At the average V1 magnetopause distance a ratio $B_{r c} / B_{d p} \approx 0.5$ is obtained. The contribution of the thermal plasma pressure in the outer magnetosphere depends on the plasma- $\beta$ adjacent to the magnetopause and is described by an additional factor: $f_{p}=$ $1+\beta$.

[10] With all considerations above the magnetopause standoff distance in units of the equatorial radius is given by:

$$
r_{m p, 0}=\left(C \frac{\cos ^{2} \psi B_{e q}^{2}}{2 \mu_{0}} \frac{1}{p_{d y n}}\right)^{1 / 6} \text { where } C=\frac{f^{2} f_{r}^{2} f_{p}}{k}
$$

where $B_{e q}$ is the magnetic field on surface equator $\left(B_{e q}=\right.$ 0.21 Gauss [Smith et al., 1980; Ness et al., 1981; Dougherty et al., 2005]) and where $r_{m p, 0}$ is in units of $R_{S}$.

[11] Strictly speaking, no magnetopause distance has been observed with the dynamic pressure of the solar wind known simultaneously. Hence, we have chosen three calibration models. Calibration model 1 considers only Saturn's magnetic dipole field and the CF-currents. For the V1 inbound pass the predicted solar wind dynamic pressure for the averaged magnetopause location requires a considerable drop in dynamical pressure from its last value in the solar wind [Bridge et al., 1981], while the spacecraft crossed the magnetosheath. For the intermediate model 2 the ring current and a plasma- $\beta=1$ were used. The high plasma- $\beta=11.1$ during the Titan encounter from V1 and considerations from Lanzerotti et al. [1983] serve as a base for this assumption. The calibration factor for model $3\left(C_{3}\right)$ is obtained by assigning to the V1 magnetopause distance an increased dynamic pressure $\left(p_{d y n, 3}=\left(p_{d y n, 2}-p_{d y n, 1}\right)+\right.$ $\left.p_{d y n, 2}\right)$, where $p_{d y n, 1}$ and $p_{d y n, 2}$ are the dynamic pressures estimated for models 1 and 2 (see Table 1 ). The results of Slavin et al. [1985] agree very well with the case of lowest internal pressure.

[12] The shape of the magnetopause is derived from the results of the ME95 model [Maurice and Engle, 1995]. For different subsolar standoff distances the shape of the magnetopause in the equatorial plane is extracted and used for a fit onto a more manageable function, presented by Shue et al. [1997]: $r_{m p}(\theta)=r_{m p, 0}(2 /(1+\cos \theta))^{\alpha}$. With fixed $r_{m p, 0}$ the flaring parameter $\alpha=\alpha\left(r_{m p, 0}, \theta\right)$ has been obtained by fitting this relation to the results of the ME95 model. The result is a less blunt magnetopause shape than derived by Slavin et al. [1985], which also has been proposed by Hansen [2001].

\subsection{Bow Shock Models}

[13] Saturn's magnetosheath thickness depends on the plasma parameters of the solar wind, including the strength and the orientation of the magnetic field [Farrugia et al., 1998]. For simplicity the angle between the magnetic field and sun direction is assumed here to be $90^{\circ}$, according to an

Table 2. Extrapolated Solar Wind Conditions at Saturn ${ }^{\text {a }}$

\begin{tabular}{lcccc}
\hline & Pioneer 11 & Voyager 1 & Voyager 2 & Ulysses \\
\hline $\mathrm{n}$ & 8484 & 3244 & 7846 & 9641 \\
$p_{d y n}$ mean, $\mathrm{nPa}$ & 0.028 & 0.016 & 0.021 & 0.034 \\
$p_{d y n}$ median, $\mathrm{nPa}$ & 0.028 & 0.017 & 0.020 & 0.022 \\
$M_{f}$ mean & 18.1 & 14.13 & 13.9 & 15.1 \\
$M_{f}$ median & 15.1 & 12.04 & 11.3 & 13.5 \\
\hline
\end{tabular}

${ }^{\mathrm{a}} M_{f}$ is the fast magnetoacoustic Mach number. 
Table 3. Radial Dependance of Solar Wind Parameters From Slavin and Holzer [1981]

\begin{tabular}{lcc}
\hline \multicolumn{1}{c}{ Parameter } & & Scaling \\
\hline Solar wind speed & $v_{s w}$ & $r^{0}$ \\
Proton density & $n_{p}$ & $r^{-2}$ \\
Magnetic field strength & $B$ & $r^{-1} \sqrt{2 r^{-2}+2}$ \\
Proton temperature & $T_{p}$ & $r^{-2 / 3}$ \\
\hline
\end{tabular}

ideal Parker spiral. The standoff distance in units of the subsolar magnetopause distance is given by a theoretical relation [Petrinec and Russell, 1997]:

$$
r_{b s, 0}=r_{c}\left(\frac{r_{m p, 0}}{r_{c}}+0.8 \frac{2 \frac{\rho_{\infty}}{\rho}}{(1+\gamma)\left(1-\frac{\rho_{\infty}}{\rho}\right)}\right)
$$

where $r_{c}$ is the radius of curvature of the magnetopause. Based on the original findings of Spreiter et al. [1966] this relation has been extended for lower Mach number [Petrinec and Russell, 1997] and arbitrary magnetopause shapes [Farris and Russell, 1994]. The magnetopause shape is defined by $r_{c}$, which can be obtained approximately from the terminator distance and the nose standoff distance [Farris and Russell, 1994]. The density ratio $\rho_{\infty} / \rho$ at the bow shock must fulfill the magnetohydrodynamic RankineHugoniot relations and is solved using the method of Petrinec and Russell [1997].
[14] The shape of the bow shock is described by a hyperboloid, defined by its subsolar standoff distance and the asymptotic Mach cone angle. Since the Mach angle does not correlate with the subsolar distance, a $1 / M$ relation between subsolar standoff distance and Mach angle is assumed, linking a large nose distance to a low Mach number, and typical Mach numbers to average standoff distances of the bow shock.

\section{Solar Wind}

[15] Plasma data from Pioneer 11, Voyager 1, Voyager 2 and Ulysses have been used to determine the solar wind conditions at Saturn. The plasma parameters at an average Saturn position have been derived from the spacecraft position by scaling laws [Slavin and Holzer, 1981, see Table 3]. The valid use of these scaling laws requires that the difference in the heliographic radial distance does not exceed 1 AU. An exception has been made for Ulysses, which has been chosen to gather information from a different period in the solar cycle. The Ulysses plasma data considered for this work is restricted to the latitude of Saturn's orbital motion. The missing electron temperature has been obtained by the relation of Sittler and Scudder [1980] assuming its validity for heliographic distances greater than $5 \mathrm{AU}$. An overview of the plasma data is shown in Table 2. There is no evidence for a bimodal
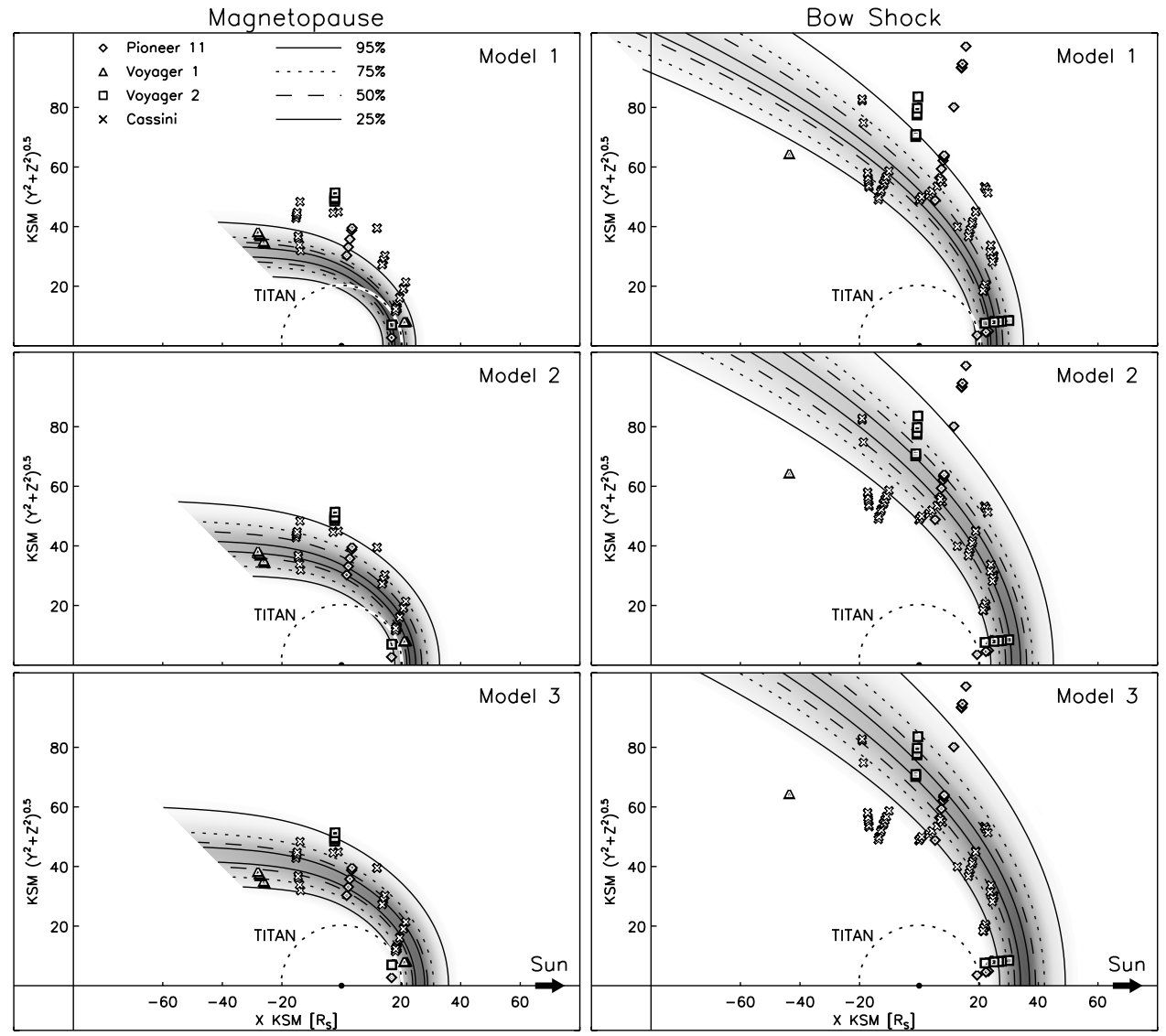

Figure 1. Probability distribution of magnetopause and bow shock locations for different calibration models. At a given Saturnian local time the magnetopause has a probability of $95 \%$ to be located between the two solid lines. The probability of being outside the solid lines is $2.5 \%$ on both sides (other line pairs defined analogously). The gray shading gives probability density. Comparison with spacecraft observations is also presented. See key for spacecraft at upper left. 
distribution in the dynamical pressure. The monomodal distribution of magnetopause distances obtained is in contrast to a study by Joy et al. [2002], which presents evidence for a bimodal distribution in distances. Table 3 .

\section{Results and Conclusions}

[16] The probability distribution is calculated in bins of the size $1 R_{S}$ for each boundary. Along radial lines with a defined local time the probability distribution in distance has been obtained by appropriate scaling of the surface models used for the bow shock and magnetopause. The result for each calibration model is shown in Figure 1 along with the Pioneer-Voyager [Wolfe et al., 1980; Ness et al., 1981, 1982] and Cassini observations on initial sunward orbits until February 2005. A cylindrical coordinate system is used. For application to Titan, we consider the subset of the distributions limited to the local time interval from 03:00 through noon to 21:00.

[17] A formal $\chi^{2}$-test confirms the results of our visual inspection that magnetopause model 2 is the best one. However, the significance of the test is limited, because the number of MP-observations is still relatively small (48) and the boundary crossings are not always statistically independent. For the bow shock model the test with 79 crossings is of limited use. The full spread of bow shock observations cannot be reproduced by a single model. Therefore, we expect these models to be less accurate for large distances from the noon sector and extreme solar wind conditions. The difference in statistical significance between magnetopause model 2 and 3 is very small. But since a significance of model 1 can be ruled out, we conclude that the magnetosphere is probably more expanded than that derived in earlier studies. As a direct result of our work, close near noon flybys at Titan by Cassini will take place in the magnetosphere for average solar wind conditions in contrast to the results of Slavin et al. [1985]. But since our probabilistic approach is idealized and a single model cannot describe all the observations (especially the PioneerVoyager encounters) an immersion of Titan in solar wind plasma during a Cassini flyby cannot be completely ruled out.

[18] Acknowledgment. S.H. and F.M.N acknowledge support by DLR, Germany.

\section{References}

Backes, H., et al. (2005), Titan's magnetic field signature during TA encounter of Cassini, Science, 308(5724), 992-995.

Behannon, K., R. Lepping, and N. Ness (1983), Structure and dynamics of Saturn's outer magnetosphere and boundary regions, J. Geophys. Res., 88(A11), 8791-8800

Bridge, H. S., et al. (1981), Plasma observations near Saturn: Initial results from Voyager 1, Science, 212, 217-224.

Connerney, J. E. P., M. H. Acuña, and N. F. Ness (1983), Currents in Saturn's magnetosphere, J. Geophys. Res., 88(A11), 8779-8789.

Dougherty, M., et al. (2005), Cassini magnetometer observations during Saturn orbit insertion, Science, 307, 1266-1270.
Farris, M., and C. Russell (1994), Determing the standoff distance of the bow shock: Mach number dependence and use of models, J. Geophys. Res., 99(A9), 17,681-17,689.

Farrugia, C., H. Biernat, and N. Erkaev (1998), The effect of the magnetopause shapes of Jupiter and Saturn on magnetosheath parameters, Planet. Space Sci., 46(5), 507-514.

Hansen, K. (2001), MHD simulation of the magnetospheres of Jupiter and Saturn: Application to the Cassini mission, doctoral thesis, Univ. of Mich., Ann Arbor, Mich.

Joy, S., M. Kivelson, R. Walker, K. Khurana, C. Russell, and T. Ogino (2002), Probabilistic models of the Jovian magnetopause and bow shock locations, J. Geophys. Res., 107(A10), 1309, doi:10.1029/ 2001JA009146.

Lanzerotti, L., C. Maclennan, R. Lepping, and S. Krimigis (1983), On the plasma conditions at the dayside magnetopause of Saturn, Geophys. Res. Lett., 10(12), 1200-1202.

Maurice, S., and I. Engle (1995), Idealized Saturn magnetosphere shape and field, J. Geophys. Res., 100(A9), 17,143-17,151.

Ness, N. F., M. H. Acuña, R. P. Lepping, J. E. P. Connerney, K. W. Behannon, L. F. Burlaga, and F. M. Neubauer (1981), Preliminary results at Saturn from the magnetic field experiment on Voyager 1, Science, 212, $211-217$.

Ness, N., M. Acuña, K. Behannon, L. Burlaga, J. Connerney, R. Lepping, and F. Neubauer (1982), Magnetic field studies by Voyager 2: Preliminary results at Saturn, Science, 215, 558-563.

Petrinec, S., and C. Russell (1997), Hydrodynamic and MHD Equations across the bow shock and along the surfaces of planetary obstacles, Space Sci. Rev., 79, 757-791.

Scarf, F., W. Kurth, D. Gurnett, H. Bridge, and J. Sullivan (1981), Jupiter tail phenomena upstream from Saturn, Nature, 292, 585, 586.

Shue, J., J. Chao, H. Fu, C. Russell, P. Song, K. Khurana, and H. Singer (1997), A new functional form to study the solar wind control of the magnetopause size and shape, J. Geophys. Res., 102(A5), 94979511.

Sittler, E. C., and J. D. Scudder (1980), An empirical polytrope law for solar wind thermal electrons between 0.45 and $4.76 \mathrm{AU}$ : Voyager 2 and Mariner 10, J. Geophys. Res., 85(A10), 5131-5137.

Slavin, J., and R. Holzer (1981), Solar wind flow about terrestrial planets: 1. Modelling bow shock position and shape, J. Geophys. Res., 86(A13), $11,401-11,418$

Slavin, J., E. Smith, P. Gazis, and J. Mihalov (1983), A Pioneer-Voyager study of the solar wind interaction with Saturn, Geophys. Res. Lett., 10(1), 9-12.

Slavin, J., E. Smith, J. Spreiter, and S. Stahara (1985), Solar wind flow about the outer planets: Gas dynamic modelling of Jupiter and Saturn bow shocks, J. Geophys. Res., 90(A7), 6275-6286.

Smith, E. J., et al. (1980), Saturns magnetosphere and its interaction with the solar wind, J. Geophys. Res., 85(A11), 5655-5674.

Spreiter, J., A. Summers, and A. Alksne (1966), Hydromagnetic flow around the magnetosphere, Planet. Space Sci., 14, 223-253.

Voigt, G. (1995), Magnetospheric configuration, in Handbook of Atmospheric Electrodynamics, edited by H. Volland, 2nd ed., CRC Press, Boca Raton, Fla.

Wolf, D. A., and F. M. Neubauer (1982), Titan's highly variable plasma environment, J. Geophys. Res., 87, 881-885.

Wolfe, J., J. Mihalov, H. Collard, and D. McKibbin (1980), Preliminary results on the plasma enviroment of Saturn from the Pioneer 11 plasma analyzer experiment, Science, 207, 403-407.

N. Achilleos and M. K. Dougherty, Space and Atmospheric Physics, Imperial College London, Prince Consort Road, London SW7 2AZ, UK.

S. Hendricks, Alfred Wegener Institute for Polar and Marine Sciences, Building F, Bussestr. 24, D-27570 Bremerhaven, Germany. (shendricks@) awi-bremerhaven.de)

F. M. Neubauer, Institute for Geophysics and Meteorology, University of Cologne, Albertus-Magnus-Platz, D-50923 Cologne, Germany.

C. T. Russell, Institute of Geophysics and Planetary Physics, University of California, Los Angeles, CA 90024-1567, USA. 